Voix et Images

voixetimages

\title{
Les poètes à bras raccourcis
}

\section{André Brochu}

Volume 29, numéro 2 (86), hiver 2004

Le laboratoire de l'écriture : manuscrits et variantes

URI : https://id.erudit.org/iderudit/008780ar

DOI : https://doi.org/10.7202/008780ar

Aller au sommaire du numéro

Éditeur(s)

Université du Québec à Montréal

\section{ISSN}

0318-9201 (imprimé)

1705-933X (numérique)

Découvrir la revue

\section{Citer cet article}

Brochu, A. (2004). Les poètes à bras raccourcis. Voix et Images, 29(2), 169-171.

https://doi.org/10.7202/008780ar d'utilisation que vous pouvez consulter en ligne.

https://apropos.erudit.org/fr/usagers/politique-dutilisation/ 


\title{
P O ÉSIE
}

\author{
Les poètes à bras raccourcis \\ $+++$
}

\author{
ANDRÉ BROCHU \\ Université de Montréal
}

Au Québec, une excellente façon de se faire des ennemis est d'être né ailleurs. Une autre est de croire que les traditions culturelles et littéraires existent en Europe et dans le monde depuis longtemps, et que celle qui s'élabore ici depuis quelques décennies gagnerait à s'aligner un peu sur les valeurs universelles. À s'aligner aussi sur une pratique de la langue qui soit bien française (ce qui n'interdit pas le souci de l'expression locale). Enfin, ces deux premières conditions restant insuffisantes, il convient d'être polémiste. Le polémiste est quelqu'un qui choque, souvent parce qu'il dit vrai et que, assenant les vérités sans ménagement, il semble provoquer le lecteur pour le futile plaisir de heurter sa sensibilité.

Jean-Pierre Issenhuth ${ }^{1}$ remplit bien les trois conditions que j'ai énumérées. Né en France, québécois depuis des lustres, il s'est bâti une culture remarquable, a fait paraître quelques poèmes inspirés et d'une grande rigueur formelle ${ }^{2}$, et il a publié, dans quelques revues et dans Le Devoir, des chroniques qui lui ont valu une inimitié quasi générale. Ces chroniques portent indifféremment sur la poésie d'ici et d'ailleurs.

Cette inimitié se comprend, car Issenhuth s'en prend à tout ce qui, en poésie québécoise, relève de la formidable institution qui s'est créée au cours des trente ou quarante dernières années. Il y a d'abord l'écriture institutionnelle justement, par exemple celle des formalistes, post-formalistes et féministes qui s'est développée autour de La Barre du jour, de La Nouvelle Barre du jour et des Herbes rouges, et qui implique une pratique du langage à laquelle notre censeur est particulièrement allergique. Mais il y a aussi tous les rituels mis sur pied, les lectures publiques, les tournées locales et à l'étranger, surtout ce colossal festival annuel de Trois-Rivières auquel Góngora, Hölderlin auraient de loin préféré, dit-il, le festival de la galette de sarrasin qui se tient parallèlement, à Louiseville (265).

La galette de sarrasin, en effet, cumule plusieurs vertus qui la rendent aimables à Issenhuth: elle est petite, elle est nature (malgré un brin de cuisson), elle vous livre tout un monde de sensations visuelles, olfactives, gustatives, voire tactiles en un seul régal sans prétention. Elle est simple, et telle est sans doute la première qualité du bon poème.

1 Jean-Pierre Issenhuth, Le petit banc de bois (lectures libres 1985-1999), Montréal, Trait d'union, 2003, 442 p. 2 Id., Entretien d'un autre temps, Montréal, l'Hexagone, 1981, 76 p. 
«Ni simplet, ni simpliste, ni indigent: simple» (169), écrit-il à propos d'un poème de René Char, «Le martinet», où l'on ne retrouve heureusement ni la «panoplie surréaliste» ni la lourde écriture de «patte d'éléphant» coutumière du poète. Un poème tout centré sur un petit être familier, quel bonheur! «La cocotte», de la Québécoise Charlotte Melançon, qui n'a publié que quelques vers dans les revues, suscite, à bon droit du reste, une admiration infinie. On comprend que le divan, mot par lequel les Persans désignaient par métonymie un recueil de poèmes, revête la forme réduite du "petit banc de bois» pour désigner ce recueil de chroniques. Le titre s'inspire en effet du siège où Rina Lasnier s'asseyait à l'écart de tous pour rêver le monde et le poème ${ }^{3}$. Ce faisant, la poète s'installait dans un "point» qui, pour un Maurice de Guérin, établit le «contact entre la nature et nous» et rend ainsi possible l'accès à la beauté et à Dieu (109). Issenhuth, comme Lasnier, comme Guérin, est un esprit religieux. Le micro-objet, élu par l'inspiration et choisi, de préférence, dans la nature, est comme une signature de l'éternel.

La poésie selon Issenhuth exclut «l'esprit et les procédés de groupe, la logomachie, la logorrhée, l'amphigouri, l'abstraction, les généralités » (302). Dans le domaine québécois, quelques auteurs échappent, en partie du moins, à ces défauts. Issenhuth ne ménage pas son admiration pour Nelligan, dont il permet la relecture en louant des vers que d'aucuns auraient jugés banals ( «Je suis toujours petit pour elle, quoique grand») et en négligeant les plus flamboyants. Admiration extrême pour Rina Lasnier, «le plus grand poète québécois vivant» (le jugement est de 1991), pourtant éloignée souvent de la simplicité, mais capable de tout, du minuscule et du grandiose - le minuscule est sans doute, ici, primordial. Admiration pour Saint-Denys Garneau, chez qui Pierre Nepveu décèle indûment de la prose, ainsi que, à un moindre degré, pour Alphonse Piché, dont l'âpreté naturaliste émeut. Et puis, dans les générations plus récentes, trouvent grâce aux yeux d'Issenhuth des poètes comme Pierre DesRuisseaux, Gilles Cyr (dont la renommée actuelle doit beaucoup à ces commentaires), Denys Néron (il le fait découvrir aux critiques), Marie Uguay, Jacques Brault, Pierre Nepveu (Mahler et autres matières), Jean-Marc Fréchette (dont Issenhuth est l'un des rares commentateurs à reconnaître la valeur exceptionnelle), Charlotte Melançon et son mari, Robert (qui est aussi un ami du chroniqueur), François Hébert (Lac noir). Sans doute, le bref poème centré sur un objet ponctuel de la nature, conjurant ainsi l'approximation et la généralité tout en s'ouvrant à la suggestion, y est-il fortement représenté.

Dans cet ensemble de lectures favorables, on constate une certaine diversité. Impossible de reprocher à l'usager du Petit banc de bois de mépriser en bloc la littérature du Québec. En revanche, beaucoup de poètes importants et parfois célébrés en dehors de nos frontières, reconnus même par des anthologies de maisons d'édition importantes (je pense à la collection «Poésie» de Gallimard, qui accueille quelques poètes québécois récents dans l'Anthologie de la poésie française du XX siècle, t. 2) font l'objet des brocards humiliants d'Issenhuth, au nom d'une conception de la poésie qui a certes le mérite de l'exigence et de la rigueur, mais qui manifeste aussi son côté étriqué quand elle refuse systématiquement les grands courants de la modernité.

Une des têtes de Turc du chroniqueur est Claude Beausoleil, que beaucoup d'universitaires se plaisent aussi à considérer de haut. Il voit en lui du «bavardage» et de l'«inflation», mais lui reconnaît tout de même «[du] tempérament, [de l']énergie, [du]

$+++$

3 En quatrième de couverture, l'auteur explique le titre de son ouvrage en référence avec Rina Lasnier. 
souffle» (250). Au polémiste, on ne peut demander plus de concessions à la vérité - que, du reste, il prétend servir. Il lui importe avant tout de frapper les imaginations, de prendre le contre-pied des facilités communes.

Et dans la mesure où Issenhuth nous met en garde, avec souvent beaucoup d'àpropos, contre la facilité et une attitude de plats consommateurs en poésie, la lecture de son livre est tout à fait recommandable. Il y défend de grandes exigences, et nous montre qu'elles sont au travail dans les œuvres poétiques importantes de notre siècle et des siècles passés, qu'il s'agisse de Mandelstam, d'Emily Dickinson ou, à l'occasion, de poètes très peu connus encore, mais que sa curiosité créatrice lui aura fait découvrir. 